\title{
Fuzzy Based Bridge Structural Health Rating of Existing Bridges using Accelerometer Sensors: Case of Zamora Bridge
}

\author{
John Victor J. Lau, Charmaine Aila Mai L. Bassig , Earl Justin C. Celino, and John Paul D. Carreon \\ Mapúa University, Muralla St., Intramuros, Manila, PHILIPPINES
}

\begin{abstract}
This study presents the development of a fuzzy-based procedure for condition assessment and bridge rating in structural health monitoring of existing bridges. A sample bridge, Zamora Bridge, was assessed to determine its structural health through fuzzy-based analysis utilizing accelerometer sensors installed in the bridge. Sensors were installed on superstructure and substructure components to record the three-dimensional accelerations of each element. Accelerations were then compared to the designed acceleration of the member to obtain structural ratings. Using fuzzy logic, the resultant rating set for the bridge has been evaluated based on specific ratings and importance factor for elements of the bridge. The final rating for the bridge can be evaluated by converting the collective fuzzy rating that defines the membership value. Comparison between the data of fuzzy based analysis for structural health assessment and visual bridge inspection for condition rating verified the reliability of the findings of this study.
\end{abstract}

\section{Introduction}

There are 8,131 permanent bridges in the sixteen regions of the Philippines according to the Department of Public Works and Highway (DPWH) as of December 2015. Bridges connect barangays, cities and regions to provide goods and mobility thus should be always functional especially during critical conditions in the area. Visual inspection is employed by DPWH to assess the structural integrity of existing bridges. This takes time to complete the assessment of the existing bridges. As indicated by various codes, assessment of existing bridges was developed; deterioration and performance were accounted [1].

In one of the earliest accounts in bridge condition assessment, bridge elements were considered from substructure, superstructure and deck [2]. Assessment was carried out not only to identify the structural health but also detect damages for failure prevention [3]. With the importance of the bridge in mobility, bridges have to be maintained and assessed frequently with reliability. This study aimed to develop a fuzzy-based structural health assessment of bridges utilizing readings from sensors installed at key structural members.

The study covered the development of a systematic process and program that will assess structural health of bridges using fuzzy based analysis. As such, this study was limited to the analysis and interpretation of data collected from installed accelerometer sensors on and the design specifications of Zamora bridge; the establishment of membership functions; the incorporation of relative importance factors; and, the development of a program for the structural health monitoring.

The study covered the development of a systematic process and program that will assess structural health of bridges using fuzzy based analysis. As such, this study was limited to the analysis and interpretation of data collected from installed accelerometer sensors on and the design specifications of Zamora bridge; the establishment of membership functions; the incorporation of relative importance factors; and, the development of a program for the structural health monitoring.

\section{Fuzzy Logic}

Visual inspection techniques were the primary methods used to evaluate the condition of the majority of bridges. These subjective assessments cause impact on the safety and management of a bridge [4].

The bridge evaluation and remaining service life prediction processes were conducted in order to efficiently maintain existing bridges, it was based on the results of periodical visual inspections [5]. Conventional methods for crack detection were applied by experienced inspectors who mark the cracks manually and read the width of the cracks with their naked eyes [6,7]. 


\section{Fuzzy Logic}

Fuzzy logic covers the shortcomings of traditional Boolean logic which is currently used for visual bridge condition assessment, and therefore can provide more accurate results. Fuzzy logic is an evaluation process wherein statements or values are represented in varying degrees of truthfulness [8] as opposed to Boolean Logic which is confined to either a true $(1)$ or a false $(0)$ [9]. In Fuzzy logic, there exist degrees such as partially true, slightly false, which can be represented as real numbers between 1 and 0 .

Fuzzy logic is based on the concept of the fuzzy set. These are sets without a clearly defined boundary [10]. Contradictory to a fuzzy set, a classical set is one with clearly defined boundaries. These cases cannot be possible in classical sets, therefore, to define such cases, the concept of fuzzy sets was introduced.

\section{Bridge Condition Assessment}

Utilizing fuzzy logic concepts, a condition rating which evaluates a reinforced concrete bridge's strength and serviceability condition can be formulated [11]. Sasmal et al. proposed a systematic approach for the condition rating of existing bridges through combining fuzzy mathematics with techniques from eigenvector-based priority-setting. In this study however, a simplified fuzzy concept was applied using fuzzy weighted average approach.

\section{Research Setting and Design}

The study considered the structural components of the bridge where accelerometer sensors were installed, these locations shall be referred to as nodes. The structural components of the bridge, its National Bridge Elements (NBEs), is divided into three major components, namely: deck, superstructure, and substructure. Each component was further divided into several elements. For the case study, analysis covered select girders in the superstructure, and select piers in the substructure of the chosen sample bridge, Zamora bridge.

The Padre Jacinto Zamora Bridge spans about 410 meters, linking Paco and Pandacan, Manila, Philippines and is a reinforced concrete bridge built in the 1960's and became operational in 1963. Periodic inspection was done on the bridge through the years by bridge inspectors of the DPWH.

Through analysis of readings of installed accelerometer sensors on the bridge, the structural health of the Zamora Bridge is determined. The sensors provide real-time structural assessment of Zamora bridge by translating sensor acceleration outputs into more comprehensible standards used by the DPWH, to hasten bridge maintenance proceedings and avoid greater losses in the case of failure. Data necessary for the study were taken from the SMART Bridge Project and the latest available bridge inspection data from the DPWH. Statistical analyses and quantitative comparison of values were utilized to produce a well- grounded basis for the structural health monitoring system of Zamora bridge.

The Smart Bridge sensors installed in Zamora bridge were placed on the columns and on the interior face of the concrete girders of the bridge for the reason that these members are easily accessible and are subject to deflection.

Each interior girder was installed with three (3) sensors: one at the midspan of the girder, and two sensors placed $2 \mathrm{H}$ from each support, wherein $\mathrm{H}$ was the overall depth of the girder. Sensors were placed on the midspan since it is where the highest deflection was most likely to occur and was the theoretical weakest point of the member.

The sensors record 100 samples per second of acceleration of the member with respect to the $\mathrm{x}, \mathrm{y}$, and z- axes respectively of each sensor.

Adopted from a study done in Indiana and its neighboring states, where a survey was conducted with bridge engineers and inspectors, the structural importance for each membership function was established [12]. In this study, the scale used was adjusted into four classifications: good, fair, poor and bad, which are the membership functions used in this study.

Table 1 shows the converted importance factors via interpolation for each classification to be multiplied to the membership functions for each node.

Table 1. Element Importance Factors

\begin{tabular}{|l|l|l|l|l|}
\hline & Bad & Poor & Fair & Good \\
\hline Pier & 1.0000 & 0.8700 & 0.7000 & 0.4300 \\
\hline Girder & 1.0000 & 0.9500 & 0.8500 & 0.5800 \\
\hline Superstructure & 1.0000 & 0.8867 & 0.7633 & 0.4700 \\
\hline Substructure & 1.0000 & 0.6333 & 0.3167 & 0.1000 \\
\hline
\end{tabular}

The designed acceleration of the members taken from STAAD is converted to LSB to correspond for each of the membership function automatically.Fuzzified data served as inputs for the fuzzy based mathematical analysis to determine the fuzzy rating of the entire bridge. The fuzzified data of each of the member had a single value for every membership function. Thus, the resulting data was a one-by-four matrix for each of the axes.

A $1 \times 4$ matrix was formed in Eq. 1 when the mean acceleration in the $\mathrm{X}$-axis is determined in the different MFs. The fuzzified data for the 3-dimensional axes were 
then combined into a larger matrix that has a dimension of $3 \times 4$ as shown in Eq. 2 .

\section{$\left[\begin{array}{llll}0.7445 & 0.255 & 0 & 0\end{array}\right]$}

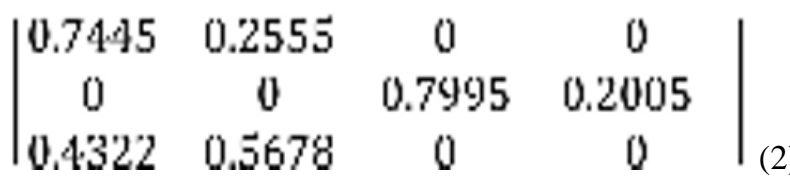

Eq. 2 was multiplied with a weight factor. Each element of the matrix represents the relative importance of each axis. The matrix was normalized resulting to Eq. 3 which shows the membership values for all the MFs considering actual acceleration of the member on 3D analysis.

\section{$\left[\begin{array}{llll}0.3992 & 0.2744 & 0.2665 & 0.0668\end{array}\right]$}

The relative importance factors were adopted [8]. The members were then classified to either superstructure elements or substructure elements. Piers were considered as substructure elements while girders were considered as superstructure elements which result to a 1 x 5 matrix.

For the structural health rating of Zamora bridge, a scale of $0 \%$ to $100 \%$ was used. A rating of $100 \%$ would mean that the bridge was in the perfect condition; $0 \%$ in the worst condition. The rating of good falls under $75 \%$ to $100 \%$, fair for $50 \%$ to $75 \%$, poor for $25 \%$ to $50 \%$, bad for lower than $25 \%$.

The resulting overall rating for the Zamora bridge is 0.538433 or $53.84 \%$ which is in the range of $50 \%$ to $75 \%$ that corresponds to a fair condition rating for Zamora bridge. The results were then compared to the latest Bridge inspection results of the Zamora Bridge performed by the DPWH showed that the Zamora bridge was given a fair condition rating.

Although the results obtained from the program developed were the same with the result of the bridge inspection, further statistical analysis for the percentiles to be used in the ratings to optimize accuracy is necessary.

Simulations were done with the program using varying percentile ranges for the membership functions. Including the membership function established in the study, mean percent increase or decrease in the magnitudes of the accelerations were simulated to determine the percent change in acceleration for the rating of Zamora bridge to change. Table 2 illustrates the bridge structural health rating of Zamora bridge using varying percentiles for the membership functions using the same sample data taken from the SMART Bridge sensors.
Table 2. Zamora Bridge Structural Health Rating using Membership Functions (MF)

\begin{tabular}{|c|c|c|c|c|}
\hline $\begin{array}{c}\mathrm{MF}_{\mathrm{Good}} \\
(\mathrm{LSB})\end{array}$ & $\begin{array}{c}\mathrm{MF}_{\text {Fair }} \\
(\mathrm{LSB})\end{array}$ & $\begin{array}{c}\mathrm{MF}_{\text {Poor }} \\
(\mathrm{LSB})\end{array}$ & $\begin{array}{c}\mathrm{MF}_{\mathrm{Bad}} \\
(\mathrm{LSB})\end{array}$ & $\begin{array}{c}\text { Structural } \\
\text { Health } \\
\text { Rating }\end{array}$ \\
\hline $0 \%$ & $90 \%$ & $99 \%$ & $100 \%$ & $\begin{array}{c}59.3942 \% \\
(\text { Fair })\end{array}$ \\
\hline $0 \%$ & $80 \%$ & $98 \%$ & $100 \%$ & $\begin{array}{c}53.8433 \% \\
(\text { Fair })\end{array}$ \\
\hline $0 \%$ & $70 \%$ & $97 \%$ & $100 \%$ & $\begin{array}{c}50.2792 \% \\
\text { (Fair) }\end{array}$ \\
\hline
\end{tabular}

The peak values for the membership functions were adjusted to different percentiles of the design accelerations based on the STAAD file of Zamora bridge. In the study, the 80th and 98th percentiles were used, which results to a $53.84 \%$ rating for the bridge. Adjusting the percentile ranges for the fair and poor membership functions to the 90th and 99th percentile, respectively, results to a rating of $59.39 \%$.

This rating still falls under the fair condition rating region of $50 \%$ to $75 \%$, but was higher compared to the previous membership functions used. Considering that the same set of data was used for the simulation, this portrays that increasing the percentile ranges used in the establishment of membership functions results to a less conservative bridge structural health rating. This means that damages on the bridge had less impacts to the overall rating, and therefore yielded a higher structural health rating for the bridge.

Consequently, adjusting the rating lower, with the fair and poor membership functions at the 70th and 97th percentile, respectively, would result to a rating of $50.28 \%$. This also falls under the fair condition rating range of $50 \%$ to $75 \%$, but was lower compared to the first membership functions used.

Using membership functions with higher percentiles used for the fair and poor membership functions would result to a less conservative structural health rating and vice versa. This was illustrated in the simulations done, whereas the increase or decrease in the actual acceleration readings to yield the same structural health rating at the $25 \%, 50 \%$ and $75 \%$ mark were higher for the 90th and 99th percentiles for fair and poor membership functions compared to the 80th/98th and 70th/97th percentiles for fair and poor membership functions.

\section{Conclusion}

The study successfully developed a means to assess structural health rating of Zamora Bridge, a reinforced concrete bridge, through fuzzy-based analysis utilizing accelerometer sensors installed in bridge girders and piers. 
The structural health monitoring program is based on MATLAB software, using readings from the accelerometer sensors in terms of LSB, thus removing the need of unit conversion, as input. Assessment was based on the design and specifications of the bridge, analyzed through STAAD software, processed through fuzzy based analysis. This eliminates the subjective nature of visual inspection to assess bridge conditions. Consequently, the study also provides real-time bridge health ratings to improve currently used structural health monitoring programs.

Furthermore, the structural health monitoring program developed can be adjusted to produce more conservative assessments of bridge condition. Peaks for good, fair, poor and bad criteria can be revised through statistical analysis of projected design accelerations. Placing peak values for each criterion closer to the central value produces a more conservative analysis, whereas placing them farther from the central value produces less conservative analysis. The conservative design for the structural health monitoring program was aimed to detect damage early on so that maintenance can be conducted in advance.

\section{Acknowledgement}

The authors acknowledge the Department of Science and Technology (DOST) for funding the SMART Bridge Project where the data in this study were obtained. This project was proposed by Mapúa University. Also, the authors recognized the support of the Department of Public Works and Highways in assisting in the installation of the sensors.

\section{References}

1. S. Sasmal, K. Ramanjaneyulu, S. Gopalakrishnan and N. Lakshmanan, "Fuzzy Logic Based Condition Rating of Existing Reinforced Concrete Bridges," 2006.

2. A. B. Tee, "The application of fuzzy $m$ athem atics to bridge condition.," 1988.

3. Y W. R. Wickramasinghe, D. P. Thambiratnam, T. H. Chan and T. Nguyen, "Vibration characteristics and damage detection in a suspension bridge," Journal of Sound and Vibration, pp. 254-274, 2016.

4. Tarighat, A., \& Miyamoto, A. (2009). Fuzzy concrete bridge deck condition rating method for practical bridge management system. Expert Systems with Applications, 36, 12077-12085.

5. Emoto, H., Takahashi, J., Widyawati, R., \& Miyamoto, A. (2014). Performance evaluation and remaining life prediction of an aged bridge by $\mathrm{J}$ BMS. Procedia Engineering, 95, 65-74.

6. Dilena, M., \& Morassi, A. (2011). Dynamic testing of a damaged bridge. Mechanical Systems and Signal Processing , 25, 1485-1507.

7. Dingle, N. (2011, November 4). Artificial Intelligence: Fuzzy Logic Explained. Retrieved from Control Engineering: http://www.controleng.com/single- article/artificialintelligence-fuzzy-logicexplained/8f3478c13384a2771ddb7e93a2b6243d.ht $\mathrm{ml}$

8. Sheposh, R. (2017, January). Salem Press Encyclopedia of Science. Ipswich: EBSCO Publishing.

9. Fairhead, H. (2015, November 2). Introduction to Boolean Logic. Retrieved from I Programmer: http://www.i- programmer.info/babbages-bag/235logic-logic-everything-is-logic.html

10. Ross, T. J. (2010). Fuzzy Logic with Engineering Applications. West Sussex: John Wiley \& Sons.

11. Sasmal, S., Ramanjaneyulu, K., Gopalakrishnan, S., \& Lakshmanan, N. (2006). Fuzzy Logic Based Condition Rating of Existing Reinforced Concrete Bridges. Americal Society of Civil Engineers, 261273.

12. Tee, A., \& Bowman, M. (1988). Bridge condition assessment using fuzzy weighted averages. A fuzzy mathematical approach for bridge condition evaluation, 49-57. 